\title{
PARASITOSES EM CRIANÇAS: UMA REVISÃO BIBLIOGRÁFICA DOS CASOS NA AMERICA LATINA
}

\section{Parasites in children: a bibliographic review of cases in Latin America}

\author{
Sibelle Salvador ${ }^{1}$, Emilio Luiz Streck ${ }^{1,2^{*}}$
}

1 Curso de Especialização em Análises Clínicas - Universidade do Extremo Sul Catarinense, Criciúma, SC, Brasil.

2 Programa de Pós-Graduação em Ciências da Saúde - Universidade do Extremo Sul Catarinense, Criciúma, SC, Brasil.

\section{Endereço para correspondência:}

${ }^{*}$ Emilio Luiz Streck

Curso de Especialização em Análises Clínicas - UNESC.

Av. Universitária, 1105. Criciúma - SC - Bairro Universitário. CEP - 88806-000

E-mail: emiliostreck@gmail.com 


\section{Resumo}

Enteroparasitas são organismos que vivem no trato gastrointestinal de animais, como humanos ou espécies animais diversas, que estão diretamente ligados à condições de saneamento e higiene. São um problema de distribuição mundial, principalmente em países subdesenvolvidos afetando principalmente crianças entre 0 e 6 anos de idade, frequentadoras de centros de cuidados infantis. São a principal causa de mortalidade e morbidade em crianças menores de três anos de idade no mundo. Grande parte das enfermidades parasitárias são assintomáticas, entretanto, os sintomas mais comuns da infecção por parasitas são diarreia, anemia e desnutrição. Diversos fatores são considerados como propícios à população no que diz respeito a contaminação por parasitas, como as condições de higiene e saneamento, tratamento hídrico e desenvolvimento de políticas públicas para melhoria da qualidade de vida de populações isoladas. As parasitoses infectam crianças principalmente por conta da imaturidade do sistema imunológico e pouco cuidado com as condições de higiene. Normalmente as famílias que sofrem com casos de parasitoses nas crianças são aquelas que possuem baixa renda, moradores de áreas rurais e marginalizados, favorecendo um ciclo de pobreza. As infecções mais comuns ao redor do mundo são giardíase, criptosporidiose e a amebíase.

Palavras-chave: enteroparasitoses; América Latina.

\section{Abstract}

Enteroparasites are organisms that live in the gastrointestinal tract of animals, such as humans or other animal species, which are directly linked to sanitation and hygiene conditions. They are a problem of worldwide distribution, especially in underdeveloped countries affecting mainly children between 0 and 6 years old, attending child care centers. It is the leading cause of mortality and morbidity in children under three years of age in the world. Most parasitic diseases are asymptomatic; however, the most common symptoms of parasite infection are diarrhea, anemia, and malnutrition. Several factors are considered to be favorable to the population in terms of contamination by parasites, such as hygiene and sanitation conditions, water treatment and development of public policies to improve the quality of life of isolated populations. The parasites infect children mainly because of the immaturity of the immune system and deficient care with the hygienic conditions. Normally families suffering from parasitic cases in children are those with low income, living in rural and marginalized areas, favoring a cycle of poverty. The most common infections around the world are giardiasis, cryptosporidiosis and amebiasis.

Keywords: enteroparasitosis; Latin America. 
Enteroparasitas ou parasitos intestinais são organismos que vivem no trato gastrointestinal de animais, como humanos ou espécies animais diversas, que estão diretamente ligados à condições de saneamento e higiene ${ }^{1}$. As enteroparasitoses são um problema de distribuição mundial, principalmente em países subdesenvolvidos ou aqueles em desenvolvimento, afetando principalmente crianças entre 0 e 6 anos de idade, frequentadoras de centros de cuidados infantis ${ }^{2}$. São a principal causa de mortalidade e morbidade em crianças menores de três anos de idade no mundo ${ }^{3}$. Grande parte das enfermidades parasitárias são assintomáticas, entretanto, os sintomas mais comuns da infecção por parasitas são diarreia, anemia e desnutrição ${ }^{4}$. Os parasitas são comuns entre os agentes causadores de infecções por conta dos mecanismos de disseminação que desenvolveram, tornando-se oportunistas, aproveitando-se do estado fisiológico do hospedeiro para disseminar a infecção ${ }^{5}$.

No Brasil, segundos dados obtidos em pesquisas dos anos de 1996 a 1997, os índices de prevalência de enteroparasitoses em crianças ocupava a segunda colocação entre outras 20 doenças mais frequentes em populações que se encontram em estado de pobreza, sendo que os índices chegavam a quase $15 \%$. Nas diferentes regiões do país, os índices apresentados foram, no Nordeste, 17,4\%, no norte $15,5 \%, 13,1 \%$ no centro oeste, $11,6 \%$ na região sudeste e por fim, $5,6 \%$ na região sul ${ }^{6}$.

Em 2009, a Organização Mundial da Saúde (OMS) divulgou um estudo revelando que cerca de nove milhões de crianças morrem antes de completar cinco anos de idade, sendo associada à essas mortes, a diarreia ${ }^{7}$. Nos países em desenvolvimento, a associação com morte infantil com a ocorrência de desordens intestinais é de 2,5 milhões de mortes por ano ${ }^{7}$.

Diversos fatores são considerados como propícios à população no que diz respeito a contaminação por parasitas, como as condições de higiene e saneamento, tratamento hídrico e desenvolvimento de políticas públicas para melhoria da qualidade de vida de populações isoladas ${ }^{3}$. Esse artigo teve como objetivo reunir informações gerais sobre enteroparasitoses em países da América 


\section{Fisiopatologia}

Latina; para a realização do mesmo realizou-se busca de artigos e outros materiais científicos sobre o tema em bases de dados como Scielo e Pubmed.

\section{EPIDEMIOLOGIA DAS PARASITOSES E FATORES DE RISCO}

Os dados epidemiológicos descritos pela Organização Mundial da Saúde (OMS) não são animadores, pois estimam que cerca de um terço da população esteja parasitada e que apenas $10 \%$ dessa população apresente sintomas ocasionados pela parasitose ${ }^{5}$. Destas, cerca de 155000 pessoas morrem em decorrência de complicações causadas pela enfermidade ${ }^{8}$. Na América Latina, as infecções causadas por helmintos chega a 30\%, sendo ainda responsável por cerca de 49,9 milhões de mortes $^{8}$. Em crianças menores de cinco anos, os índices de morte e diarreia aguda são maiores, principalmente naquelas que são assistidas em centros especializados, como creches, em que surgem como surtos ou casos isolados, que vão de 6 a $250 \%$ maior ${ }^{9}$.

Em países da América Latina como a Venezuela, por exemplo, as infecções causadas por Ascaris lumbricoides é a mais importante para a saúde pública, pois a frequência da infecção, associada a morbidade tornou-se um problema de saúde pública, tendo em vista o aumento de $49 \%$ nos casos de infecção, somados ao aumento de Trichuris trichiura de $25,82 \%$, acometendo principalmente crianças ${ }^{10}$. No Brasil, a estimativa de infecção por parasitas é de $55,3 \%$ das crianças, sendo a infecção associada a desnutrição e diarreia crônica, fatores que podem interferir diretamente no desenvolvimento da criança, interferindo no desenvolvimento cognitivo e físico, podendo evoluir para óbito ${ }^{9}$.

No Uruguai, apesar das reformas nos sistemas públicos de saúde, as infecções parasitárias em crianças ainda são alarmantes, porque muitas famílias ainda vivem em comunidades afastadas, em condições precárias de higiene e limpeza, com difícil acesso a recursos como medicamentos e médicos ${ }^{8}$. Em um estudo realizado, o levantamento dos dados de infecções parasitárias em crianças foi de $37,7 \%$, dos quais o mais encontrado foi Enterobius vermicularis em $34,7 \%$ dos casos $^{8}$.

O contato mais próximo com outras pessoas em creches e casas de assistência favorecem a contaminação por parasitas em crianças assistidas. Os 


\section{Fisiopatologia}

hábitos de higiene, principalmente das mãos e a colocação de objetos na boca favorecem a disseminação de patógenos as crianças $^{9}$. Embora as doenças parasitárias sejam indicativos mundiais de condições socioeconomicas, esse problema de saúde pública também pode afetar adultos, gerando problemas gastrointestinais, perda de vitalidade e consequentemente uma lentidão no processo de desenvolvimento de tarefas. A giardíase, por exemplo, nos países em desenvolvimento, é a maior causa de diarreia em crianças, associando a problemas de desnutrição ${ }^{11}$.

As infecções causadas por Trichuris trichura acometem cerca de 400 mil crianças por ano, variando de acordo com a geografia, e onde se encontram as áreas urbanas. No Peru, as populações testadas para o estudo se concentraram em áreas urbanas de Lima e nas áreas rurais da Amazônia. As crianças são as que mais sofrem, porque a infecção envolve mecanismos de infecção e consequentemente desenvolvem processos de inflamação crônica, má absorção de nutrientes e perda de sangue. A OMS sugere que as crianças sejam tratadas com a maior rapidez possível, porém os tratamentos inconsistentes contribuem para morbidade crônica, incluindo anemia e desnutrição ${ }^{12}$.

As parasitoses infectam crianças principalmente por conta da imaturidade do sistema imunológico e pouco cuidado com as condições de higiene. Normalmente as famílias que sofrem com casos de parasitoses nas crianças são aquelas que possuem baixa renda, moradores de áreas rurais e marginalizados, favorecendo um ciclo de pobreza. As infecções mais comuns ao redor do mundo são giardíase, criptosporidiose e a amebíase ${ }^{13}$.

No Chile, os estudos com parasitoses em crianças são escassos. As deficiências higiênicas e a escassa infraestrutura sanitária, associadas a pobreza e as condições de risco naquele pais contribuem para o aumento da incidência de parasitoses nessa população. Nos últimos anos, o Chile melhorou seus indicadores sociais e com investimentos na educação e saúde, melhorando inclusive o desenvolvimento social da população e o combate a essa epidemia ${ }^{14}$.

O tipo de infecção e os tipos de parasitas que acometem essa população podem variar de acordo com a região estudada, sem distinção de raça, sexo ou estado socioeconômico. Há algumas décadas, os números absolutos de casos vem diminuindo em decorrência da frequente melhoria feita nas áreas consideradas de 


\section{Fisiopatologia}

risco, como loteamentos e áreas rurais, políticas de educação e manutenção dos serviços já feitos em setores urbanos e periféricos ${ }^{15}$.

Tratando-se de poliparasitismo, as populações rurais são as mais cometidas pois, nessas áreas, encontram-se os indivíduos sujeitos a processos contínuos de infecção e reinfecção, por conta da constante exposição aos fatores de risco das parasitoses. O calor, a umidade, a deficiência no saneamento básico em áreas rurais aumentas as chances de infecção. Nas zonas urbanas, a prevalência é de monoparasitismo ${ }^{15}$.

A frequência do poliparasitismo pode revelar as deficiências dos sistemas de saneamento e das políticas públicas para contenção dessas enfermidades. A principal via de transmissão parasitária é pela via fecal oral, sendo os mais comuns E. histolytica/dispar, Giardia intestinalis, Entamoeba coli e Ascaris lumbricoides, sendo esses parasitas excelentes bioindicadores de resistência a políticas públicas de higiene e saneamento ${ }^{16}$.

\section{DISCUSSÃO}

A prevalência de parasitas intestinais depende da exposição efetiva as formas de infecção dos patógenos, como cistos, ovos, larvas, etc., e os locais em que mais se observa a presença destes parasitos é em populações estudantis ou empregados, o qual não apresentam higiene adequada das mãos e limpeza do ambiente em que vivem, além do ambiente escolar ser susceptível a grande rotatividade de alunos e funcionários ${ }^{1}$.

É necessário que se leve em consideração as condições ambientais e sanitárias dos locais estudados, principalmente das regiões endêmicas. O Ministério da Saúde preconiza que todas as crianças acometidas por parasitoses sejam tratadas imediatamente, para evitar a disseminação das parasitoses aos demais membros da comunidade ${ }^{17}$.

A infecção pelo Ascaris lumbricoides continua sendo o principal parasita encontrado em população escolar no Brasil ${ }^{18}$. Outros parasitas, além de bactérias e vírus são comumente encontrados em crianças em idade escolar, principalmente pelas condições de higiene e classe econômica, que pode ser observada a diarreia como condição fisiológica principal das patologias gastrointestinais ${ }^{9}$. 
A diferença de classificação socioeconômica na prevalência das doenças parasitarias é relativamente maior entre a população que possui renda maior e maior informação, além de bens de consumo como televisão e geladeira ${ }^{2}$. É notório que quanto menos condição sanitária é observada nos estudos, maior a incidência de parasitoses, visto a diferença de instrução e cuidados dos pais e creches estudadas $^{10}$.

Estudos para conhecimento das regiões acometidas por infecções ou surtos de parasitoses são comuns, e populações como a infantil são os principais alvos de estudo. Os estudos utilizados nesse artigo confirmam a existência de elevada prevalência de parasitoses em toda América Latina, embora grande parte dos países tenha feito grandes investimentos na área de educação sanitária e em saneamento básico. Na Venezuela, por exemplo, as infecções parasitárias em crianças são enormes, em cidades que possuem poucos recursos e em diferentes estados, favorecendo a disseminação das infecções nas populações estudadas, visto a pouca ou nenhuma informação a respeito do assunto. As maiores incidências de parasitoses são encontradas por helmintos ${ }^{19}$.

O uso abusivo de terapias antiparasitárias por automedicação tem sido um problema para as autoridades de saúde pública, tendo em vista o controle dessas parasitoses, pois, em muitos casos, não são notificados para estudos epidemiológicos posteriores ${ }^{15}$. Grande parte dos medicamentos usados para esse fim são medicamentos com ação a protozoários, o que se presume um aumento de infecção por essa classe ${ }^{19}$.

Para que se tenha um controle e consiga melhorias nos casos de parasitoses, um exame parasitológico de fezes seria o ideal para diagnosticar e controlar 0 estado de saúde da criança, mesmo quando entra nas creches e nas casas de recreação, pois, desse modo, o controle e eficiência do combate às parasitoses será mais eficiente. Até mesmo as crianças provenientes de centros urbanizados apresentam risco de contrair doenças em creches e casas de recreação, sendo o exame indispensável a todos os frequentadores ${ }^{20}$.

A qualidade da água consumida ainda é fator determinante para a transmissão e contenção da epidemia de parasitose. A água vendida em galões é tratada, porém, os fatores de controle de qualidade do processo de produção nem sempre são seguidos, desqualificando o líquido como livre de contaminantes ${ }^{21}$. Em 


\section{Fisiopatologia}

Cuba, as avaliações feitas relativas a água foram de que a cada cinco galões de água analisados, um não possuía as propriedades adequadas para envasamento e venda, pois apresentavam contaminação por bactérias ou protozoários ${ }^{21}$.

No Chile, a distribuição geográfica das parasitoses ainda é escassa e apresenta apenas algumas regiões específicas, por exemplo, no norte do país, nas áreas urbanas e rurais apresentam uma prevalência de 50 e $80 \%$. Nas zonas urbanas, a prevalência de parasitoses em crianças foi de $55 \%$ e em zonas rurais foi de $76 \%$, no sul do país, os números do estudo foram semelhantes aos já citados ${ }^{14}$.

Em países como a Venezuela, os estudos realizados apontam que os mais afetados pelas parasitoses ainda são as crianças. Cerca de $43 \%$ das crianças estudadas apresentam idades entre 5 e 10 anos $^{21}$. Essas infecções se tornam uma ameaça a saúde pública pela falta de informação e acessibilidade, que demanda esforço das equipes de saúde para que haja mais investigações e controle, além de conscientização das diferentes populações existentes, seja na zona rural ou urbana, para que posteriormente seja possível a realização de um novo trabalho comparativo das mesmas ${ }^{15}$.

\section{CONCLUSÃO}

Conclui-se que a prevalência de parasitoses em crianças ainda é alta e indica problemas no acesso a informação e educação sanitária, exigindo um controle maior das autoridades locais para diminuição e controle das infecções, possibilitando aos infantis uma infância saudável e sem complicações concomitantes, como desnutrição e comprometimento do desenvolvimento da criança.

\section{REFERÊNCIAS}

1. Pereira EB dos S, Rodrigues SLC, Bahia-de-Oliveira GH, Coelho SVB, Barata RA. Detection of intestinal parasites in the environments of a public school in the town of Diamantina, Minas Gerais State, Brazil. Rev Inst Med Trop Sao Paulo. 2016;58(1):2-5.

2. Queiroz Gurgel R, De Sá Cardoso G, Silva ÂM, Novais Dos Santos L, Veiga De Oliveira RC. Creche: Ambiente expositor ou protetor nas infestações por parasitas intestinais em Aracaju, SE. Rev Soc Bras Med Trop. 2005;38(3):267-9. 


\section{Fisiopatologia}

3. Rebolla MF, Silva EM, Gomes JF, Falcao AX, Rebolla MVF, Franco RMB. High prevalence of Blastocystis spp. infection in children and staff members attending pulbic urban schools in São Paulo state, Brazil. Rev Inst Med Trop Sao Paulo. 2016;58(2):31.

4. Bautista L. Revista CUIDARTE. Rev Cuid [Internet]. 2015;6(1):991-8. Available from: http://www.revistacuidarte.org/index.php/cuidarte/article/view/237/483.

5. Lucero, Tarín; Álvarez, Luis; Chicue, Jeison; López, Deyirley; Mendoza C. Parasitosis intestinal y factores de riesgo en niños de los asentamientos subnormales, Florencia-Caquetá, Colombia. Rev Fac Nac Salud Pública [Internet]. 2015;33(2):171-80. Available

http://www.scielo.org.co/scielo.php?script=sci_arttext\&pid=S0120-

$386 \times 2015000200004 \&$ Ing $=$ pt\&nrm=iso\&tIng=es

6. Oliveira MC, Da Silva CV, Costa-Cruz JM. Intestinal parasites and commensals among individuals from a landless camping in the rural area of Uberlândia, Minas Gerais, Brazil. Rev Inst Med Trop Sao Paulo. 2003;45(3):173-6.

7. Donizetti E, Castro R, Cristina M, Yassaka B, Arc JD, Mascarenhas P. Enteropathogens Detected in a Daycare Center, Southeastern Brazil : Bacteria, Virus, and Parasite Research. 2015;57(1):27-32.

8. Dall P, Cantou VRK. Ascaris lumbricoides. Antiseptic. 2014;85(3):149-54.

9. Pedraza DF, Queiroz D De, Sales MC. Doencas infecciosas em criancas preescolares brasileiras assistidas em creches. Cien Saude Colet [Internet]. 2014;19(2):511-28. Available from: http://www.scielo.br/scielo.php?script=sci_arttext\&pid=S1413$81232014000200511 \&$ Ing=pt\&nrm=iso\&tlng=en

10. Leonardo J, Barroso C, Carlos J, Artigas J, Nuñez L, Pocaterra L, et al. Evaluación inmunológica de extractos de Ascaris Lumbricoides para las inmunoglobulinas IgA en el suero de individuos infectados. 2014;68(2):48-52.

11. Lima Junior OA de, Kaiser J, Catisti R. High occurrence of giardiasis in children living on a "landless farm workers" settlement in Araras, São Paulo, Brazil. Rev do Inst Med Trop São Paulo. 2013;55(3):185-8.

12. Cabada MM, Goodrich MR, Graham B, Villanueva-Meyer PG, Deichsel EL, Lopez $M$, et al. Prevalence of intestinal helminths, anemia, and malnutrition in Paucartambo, Peru. Rev Panam Salud Publica [Internet]. 2015;37(2):69-75. Available from: http://search.ebscohost.com/login.aspx?direct=true \&db=cin20\&AN=2013000673?=es \&site=ehost-live

13. Echagüe G, Sosa L, Díaz V, Ruiz I, Rivas L, Granado D, et al. Enteroparasitosis en niños bajo 5 años de edad, indígenas y no indígenas, de 


\section{Fisiopatologia}

comunidades rurales del Paraguay. Rev Chilena Infectol 2015; 32 (6): 649-57.

14. Montt SP. Desigualdad en la prevalencia de parasitosis intestinal en escolares de una escuela urbana y dos rurales de la comuna de Puerto Montt. 2016;886-93.

15. González B, Michelli E, Guilarte DV, Rodulfo H, Mora L, Gómez T. Estudio comparativo de parasitosis intestinales entre poblaciones rurales y urbanas del estado Sucre, Venezuela. Rev la Soc Venez Microbiol [Internet]. 2014;34:97-102. Available from: http://www.scielo.org.ve/scielo.php?script=sci_arttext\&pid=S131525562014000200010

16. Cabeza MI, Cabezas MT, Cobo F, Salas J, Vázquez J. Hymenolepis nana: factores asociados a este parasitismo en un área de salud del Sur de España. Rev Chil infectología [Internet]. 2015;32(5):593-5. Available from: http://www.scielo.cl/scielo.php?script=sci_arttext\&pid=S0716$10182015000600019 \&$ lng=en\&nrm=iso\&tlng=en

17. Silva MTN, Santana JV, Bragagnoli G, Marinho AM da N, Malagueño E. Prevalence of Entamoeba histolytica/Entamoeba dispar in the city of Campina Grande, in northeastern Brazil. Rev Inst Med Trop Sao Paulo [Internet]. 2014;56(5):451-4. Available

from:

http://www.scielo.br/scielo.php?script=sci_arttext\&pid=S0036-

46652014000500451\&lng=en\&nrm=iso\&tIng=en

18. Ferraz RRN, Barnabé AS, Porcy C, D'Eça Júnior A, Feitosa T, Figueiredo P de M. Parasitoses intestinais e baixos índices de Gini em Macapá (AP) e Timon (MA), Brasil. Cad Saúde Coletiva [Internet]. 2014;22(2):173-6. Available from: http://www.scielo.br/scielo.php?script=sci_arttext\&pid=S1414-

462X2014000200173\&lng=pt\&nrm=iso\&tlng=em

19. Laugart EL, García FMR, Fidel Ángel Núñez C, Peña MSR, Fundora ICM, Medina RS. Aspectos epidemiológicos de las parasitosis intestinales en niños de vegón de nutrias, Venezuela. Rev Cubana Hig Epidemiol. 2012;50(3):330-9.

20. Alfaro $\mathrm{M}$ del $\mathrm{CH}$, Mesa $\mathrm{CP}$. Parasitismo intestinal en niños de círculos infantiles en un municipio. Rev Ciencias Médicas Pinar del Río [Internet]. 2014;18(2):210-20. Available from: http://www.revcmpinar.sld.cu/index.php/publicaciones/article/view/1488/html

21. Rojas OB, Hernandez ZA. Parasitismo intestinal en niñas y niños mayores de 5 años de Ciudad Bolívar. Medisan 2013;17(10):585-91. 